\title{
Optically Generated 2-Dimensional Photonic Cluster State from Coupled Quantum Dots
}

\author{
Sophia E. Economou, ${ }^{1}$ Netanel Lindner, ${ }^{2,3}$ and Terry Rudolph ${ }^{4}$ \\ ${ }^{1}$ Naval Research Laboratory, Washington, D.C. 20375, USA \\ ${ }^{2}$ Department of Physics, Technion-Israel Institute of Technology, 32000 Haifa, Israel \\ ${ }^{3}$ Institute for Quantum Information, California Institute of Technology, Pasadena, California 91125, USA \\ ${ }^{4}$ Institute for Mathematical Sciences, Imperial College, SW7 2PG, London, United Kingdom \\ (Received 31 March 2010; revised manuscript received 23 July 2010; published 24 August 2010)
}

\begin{abstract}
We propose a method to generate a two-dimensional cluster state of polarization encoded photonic qubits from two coupled quantum dot emitters. We combine the proposal for generating one-dimensional cluster state strings from a single dot, with a new proposal for an induced conditional phase gate between the two quantum dots. The entanglement between the two dots translates to entanglement between the two photonic cluster state strings. Further interpair coupling of the quantum dots using cavities and waveguides can lead to a two-dimensional cluster sheet, the importance of which stems from the fact that it is a universal resource for quantum computation. Analysis of errors indicates that our proposal is feasible with current technology. Crucially, the emitted photons need not have identical frequencies, and so there are no constraints on the resonance energies for the quantum dots.
\end{abstract}

DOI: 10.1103/PhysRevLett.105.093601

PACS numbers: 42.50.Ex, 42.50.Hz, 78.67.Hc

Measurement-based quantum computation (MQC) is an alternative to the well-known "circuit model" of quantum computation [1]. The main idea in MQC is to robustly create, up front, a highly entangled state. Once this "cluster state" is created, which is the challenging part of this approach, only single qubit measurements are necessary to perform the actual computation. In the case of photon polarization qubits, performing single qubit rotations followed by photon number detection is easily done with high fidelity, which makes them particularly attractive for MQC. In fact, this is one of the most fault-tolerant architectures known for quantum computing [2], and is particularly tolerant to qubit losses [3], of importance for optical architectures. The creation of the initial entangled cluster state is, however, a difficult problem on which many current research efforts are focused. To date the most promising methods have involved optical interference of nearly identical photons [4], which is a probabilistic process with the stringent requirement of identical photons. More recently, Ref. [5] proposed using a periodically pumped quantum dot (QD) for the generation of a onedimensional cluster state, which, however, is not adequate for quantum computation. Our proposal here allows for direct generation of the entangled photons in two dimensions, and advances the proposal [5] toward a directly generated universal quantum computing resource.

In Ref. [5] the linear cluster state of polarization encoded photons was generated from single photon emitters with a certain level structure, such as that found in QDs. The relevant states of the QD are the two spin states $|\uparrow\rangle,|\downarrow\rangle$ of the electron along the optical axis $z$ and the two optically excited states called trions, which have total angular momentum $3 / 2$ and have spin projections along the $z$ direction of $\pm 3 / 2$ - states we denote $\left|T_{+}\right\rangle,\left|T_{-}\right\rangle$. The broken symmetry of the QD along the $z$ axis sets a preferred direction, along which the optical polarization selection rules are circularly polarized, and energetically separates the excited trion states with total angular momentum $\pm 1 / 2$ (the light-hole states) from these heavy-hole trion states. In the process of linear cluster state generation [5], the heavyhole trions are the only excited states that are populated. The main idea in [5] is to shine a periodic train of optical linearly polarized $\pi$ pulses, to an electron that is in a superposition state $|\uparrow\rangle+|\downarrow\rangle$, exciting it to a superposition of the two trion states $\left|T_{+}\right\rangle+\left|T_{-}\right\rangle$. Because QDs have large dipole moments, spontaneous emission is very fast, both compared to atoms and to the other relevant time scales in the QD dynamics, at least for very low magnetic fields. Therefore the trion will spontaneously decay to the electron state almost instantaneously upon excitation, emitting a photon of either right $(R)$ or left $(L)$ circular polarization, thereby effecting transitions $\left|T_{+}\right\rangle \rightarrow|\uparrow\rangle|R\rangle$, $\left|T_{-}\right\rangle \rightarrow|\downarrow\rangle|L\rangle$. The state of the emitted photon + spin is $|\uparrow\rangle|R\rangle+|\downarrow\rangle|L\rangle$-i.e., they are entangled as both recombination paths take place simultaneously. The remaining degrees of freedom of the system are the same, so they are factored out and omitted for brevity. Subsequent precession of $\pi / 2 \mathrm{rad}$ by the spin about a weak magnetic field oriented in the $y$ direction is performed, denoted $R_{y}(\pi / 2)$, before subjecting the dot to another pulse excitation + emission process. Repeating this protocol results in a one-dimensional entangled chain of photons.

Here we will develop an explicit, all-optical protocol for generating a two-dimensional cluster state comprised by linking two linear chains like the ones of [5] by controlled phase (CZ) gates. To do so we present a new proposal, related to that of [6], for performing an optically controlled CZ gate between two quantum dots. Taking advantage of the exchange interactions between electrons and the hole, this gate actually proves to be faster than that of [6]. 
Crucially, unlike the scheme of [6], this process is also compatible with the operation of the single-dot photonic machine guns, as it performs the optical CZ in the $z$ basis. The entangled emitters therefore generate photons which are themselves entangled. Explicitly, an entangled ladder can be created. This circumvents the need for "fusion gates" [4]. Moreover, in our approach, the photons need not be identical in frequency, so that there are no constraints on the resonance energies of the two QDs. We note that similar ideas have been explored using atomic systems [7]. The main advantage of using quantum dots is their fast emission rates and simple level structure, properties that constitute QDs attractive photon emitters [8].

The state evolution for the idealized abstract protocol is depicted in Fig. 1. For a quantum circuit logically equivalent to the protocol, see Fig. 2. For simplicity we assume that the two QDs are initialized in the spin-up state $|\uparrow\rangle|\uparrow\rangle$. This can be done by periodic circularly polarized pulses on each spin which, in combination with spontaneous emission and spin precession in the external magnetic field along the $y$ direction, will initialize the spin to the desired state [9]. With enough pulses in the pulse train, there is no need for a large magnetic field. Alternatively, one can pump both spins and then measure the emitted single photons in order to "collapse" the state. First we apply a $R_{y}(\pi / 2)$ operation on each spin yielding $(|\uparrow\rangle+|\downarrow\rangle)(|\uparrow\rangle+$ $|\downarrow\rangle)$, as in Fig. 1(a). This is followed by a CZ gate entangling the dots, $(|\uparrow\rangle|\uparrow\rangle+|\uparrow\rangle|\downarrow\rangle+|\downarrow\rangle|\uparrow\rangle-|\downarrow\rangle|\downarrow\rangle)$, producing the bond in Fig. 1(b). Immediately after this we apply the pump pulse to each dot, and the creation of the subsequent photons yields the state $(|\uparrow\rangle|R\rangle|\uparrow\rangle|R\rangle+|\uparrow\rangle|R\rangle|\downarrow\rangle|L\rangle+$ $|\downarrow\rangle|L\rangle|\uparrow\rangle|R\rangle-|\downarrow\rangle|L\rangle|\downarrow\rangle|L\rangle)$. In the circuit of Fig. 2 this is equivalent to the CNOT gates. This resulting state is equivalent to a 2-qubit cluster state, where the logical state $|0\rangle$ $(|1\rangle)$ is redundantly encoded [4] in 2 qubits as $|\uparrow\rangle|R\rangle$

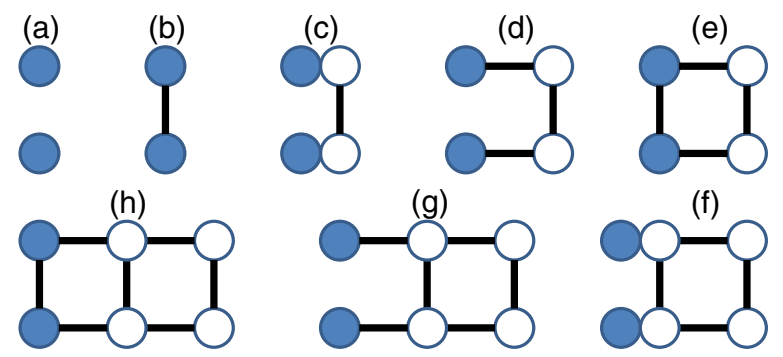

FIG. 1 (color online). Diagrams depicting the generation of the cluster state using the standard diagrammatic representations of such states. The spins are depicted as filled circles, the initial electronic state is $|\uparrow\rangle|\uparrow\rangle$. At step (a) both spins precess under $R_{y}(\pi / 2)$, at (b) the $\mathrm{CZ}$ gate is applied, at (c) a pulse excitation followed by trion decay produces photons (open circles). These procedures are then repeated, leading to the states of (d)-(h). Note that to recover the standard form of cluster states one must use a mapping where the logical qubit $|1\rangle$ state is equivalent to the photonic state $-|L\rangle[5]$.
$(-|\downarrow\rangle|L\rangle)$. Graphically such a situation is depicted with the circles for each qubit adjacent to each other, Fig. 1(c). A second $R_{y}(\pi / 2)$ on each dot pushes out the redundantly encoded qubits (i.e., creates a bond between them in the cluster state), Fig. 1(d), and we start the cycle anew.

The interdot CZ gate is implemented optically by coupling to trion states which are higher in energy than the ones used for the single-dot photon emission. These states are delocalized, i.e., the voltage bias is such that one of the electrons in these higher-energy states is tunnel-coupled, in contrast to the single-electron ground states (denoted $|B\rangle$ and $|T\rangle$ for bottom and top QD, respectively) and the lower energy trion states, which are isolated from one another and localized to their respective quantum dots. This trion mediating the interdot interaction has two electrons in the $|B\rangle$ and $|T\rangle$ (s-type) states and the third electron in the first excited ( $p$-type) orbital, which we take to be the one that is a delocalized (also called "molecular" or "extended") state, denoted $|E\rangle$. It has been shown experimentally that such a regime is feasible [10]. The hole is taken to occupy a single orbital state $|H\rangle$, which for simplicity we take to be completely confined to one QD (relaxing this assumption does not deleteriously affect the overall proposal).

With the three electrons in distinct orbital states, the spin configuration can acquire any of its allowed values by adding the three angular momenta. So, for a given orbital configuration there are a total of eight electron states (two $S=1 / 2$ doublets and one $S=3 / 2$ quadruplet) and two hole spin states, making a total of 16 states [6]. By tuning the laser appropriately, some of the states can be safely ignored due to the large $e-e$ exchange splittings (on the order of $5 \mathrm{meV}$ ) compared to the laser bandwidth.

We henceforth focus on the four $S=3 / 2$ states, which have a separable (product) form of orbital and spin states: $|3 / 2\rangle=|A\rangle|\uparrow \uparrow \uparrow, \quad| 1 / 2\rangle=|A\rangle(|\uparrow \uparrow \downarrow\rangle+|\uparrow \downarrow \uparrow\rangle+|\downarrow \uparrow \uparrow\rangle) / \sqrt{3}$,

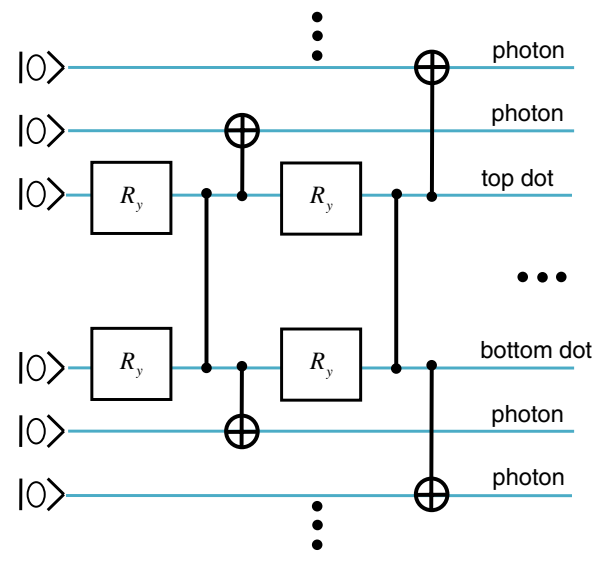

FIG. 2 (color online). A quantum circuit which is logically equivalent to the idealized evolution of the two QDs. The CZ gates correspond to the interdot coupling, the CNOT gates to photon emission, and the $R_{y}$ to precession by $\pi / 2$ around a magnetic field in the $y$ direction. 
where $\quad|A\rangle=\frac{1}{\sqrt{6}}(|T B E\rangle-|B T E\rangle-|T E B\rangle+|B E T\rangle-$ $|E B T\rangle+|E T B\rangle)$. States $|\overline{3} / 2\rangle$ and $|\overline{1} / 2\rangle$ are given by flipping all the spins.

The electron and hole in semiconductors are coupled by exchange interactions. In QDs, these are quite strong (on the order of, or stronger, than typical Zeeman energies), and they are separable into "isotropic" and "anisotropic" terms [11]. The isotropic term is much stronger-typical values of this are $0.3-0.5 \mathrm{meV}$ - so it is the leading term in our parameter regime. Its physical origin is the lack of inversion symmetry in the QD. We will ignore the anisotropic term, which originates from in-plane asymmetry (deviation of the QD cross section from a disk) and is typically small, in the order of $\mu \mathrm{eV}[11,12]$; its effects can be incorporated as standard errors in the gate.

The Hamiltonian is therefore given by $\mathcal{H}=$ $\sum_{i} \alpha_{z}\left(r_{i}, r_{h}\right) s_{i z} j_{z}$, where $\alpha_{z}\left(r_{i}, r_{h}\right)$ is an operator acting on the envelope wave functions of the electrons and the hole. The index $i$ runs over the three electrons, $r_{h}$ denotes the position of the hole, and $z$ is the growth axis. The operator $j$ acts on the hole spin, which we take to be a pseudospin, $j_{z}= \pm 3 / 2$ (i.e., ignore light-hole states). Adding and subtracting terms, we can rewrite $\mathcal{H}$ as

$$
\begin{aligned}
\mathcal{H}= & \frac{1}{3} \sum_{i} \alpha_{z}\left(r_{i}, r_{h}\right) S_{z} j_{z}+\frac{1}{3} \sum_{\substack{(i, j) \in \\
\{(12),(23),(31)\}}}\left[\alpha_{z}\left(r_{i}, r_{h}\right)\right. \\
& \left.-\alpha_{z}\left(r_{j}, r_{h}\right)\right]\left(s_{z i}-s_{z j}\right) j_{z},
\end{aligned}
$$

with $S_{z}=\sum_{i} s_{i z}$. The first term on the right-hand side of Eq. (1) conserves the total electron spin, so it only has nonzero matrix elements within the three total spin subspaces discussed above. The second set of terms has nonzero matrix elements only between different total electron spin states. Since typical values of the electron-electron exchange are about 1 order of magnitude more than typical electron-hole exchange interactions, we can ignore the total spin mixing terms and focus on the Hamiltonian $\mathcal{H} \simeq$ $\frac{1}{3} \sum_{i} \alpha_{z}\left(r_{i}, r_{h}\right) S_{z} j_{z}$, and only consider the states with $S=$ $3 / 2$ tensored with the hole state, which is an $8 \times 8$ space. The mean value of the operator $\alpha$ in state $|A\rangle|H\rangle$ is

$$
\begin{aligned}
\sum_{i}\left\langle H\left|\left\langle A\left|\alpha\left(r_{i}, r_{h}\right)\right| H\right\rangle\right| A\right\rangle & =\sum_{K=B, T, E}\left\langle H\left|\left\langle K\left|\alpha\left(r, r_{h}\right)\right| H\right\rangle\right| K\right\rangle \\
& \equiv \delta_{0}^{B H}+\delta_{0}^{T H}+\delta_{0}^{E H} .
\end{aligned}
$$

Assuming that the hole is localized in one of the two quantum dots, say the one labeled by $B$, we have $\delta_{0}^{T H}=0$. We will define the sum of the nonzero terms to be $\delta_{0}$. Now we have the operator $\mathcal{H}_{3 / 2}=\frac{\delta_{0}}{3} S_{z} j_{z}$ acting only on the spin states. Clearly, this operator is already diagonal in the basis we have chosen. Since it is invariant under the simultaneous flip of $S_{z}$ and $j_{z}$, the states are doubly degenerate. Then the eigenenergies are $E_{1}=\frac{\delta_{0}}{4}, E_{2}=\frac{\delta_{0}}{12}, E_{3}=$ $-\frac{\delta_{0}}{12}, E_{4}=-\frac{\delta_{0}}{4}$, with corresponding pairs of eigenstates $\{|3 / 2\rangle|\Uparrow\rangle,|\overline{3} / 2\rangle|\downarrow\rangle\}, \quad\{|1 / 2\rangle|\Uparrow\rangle,|\overline{1} / 2\rangle|\Downarrow\rangle\}, \quad\{|1 / 2\rangle|\Downarrow\rangle$, $|\overline{1} / 2\rangle|\Uparrow\rangle\}$, and $\{|3 / 2\rangle|\Downarrow\rangle,|\overline{3} / 2\rangle|\Uparrow\rangle\}$. The states with energy $E_{1}$ are dark. The remaining ones are optically accessible. We are interested in the states with energy $E_{4}$. These states are coupled only to the two-qubit states $|\uparrow \uparrow\rangle$ and $|\downarrow \downarrow\rangle$ by polarization $\sigma^{-}$and $\sigma^{+}$, respectively. The two-qubit states $|\uparrow \downarrow\rangle$ and $|\downarrow \uparrow\rangle$ couple to the states with $E_{2}, E_{3}$ with these polarizations. We take advantage of the energy splitting between $E_{4}$ and $E_{2}, E_{3}$ to selectively address only the twoqubit $|\downarrow \downarrow\rangle$ state and realize the $\mathrm{CZ}$ gate.

For simplicity we fix the polarization of the pulse to $\sigma^{+}$ ( behavior for the orthogonal polarization is found by flipping all the spins). If we label the dipole matrix element for transition $|\downarrow \downarrow\rangle \rightarrow|\overline{3} / 2\rangle|\Uparrow\rangle$ to be $d_{0}$, then only the triplet state $\left|T_{+}\right\rangle$couples to the excited state $|\overline{1} / 2\rangle|\Uparrow\rangle$ with dipole strength $\sqrt{\frac{2}{3}} d_{0}$. Given these three transitions, we can implement the CZ gate by acting with a resonant $2 \pi$ pulse on the $|\downarrow\rangle$ state and avoid coupling to the other transitions. Very recently, similar entangling phase gates have been experimentally realized in a quantum dot molecule [13].

We now turn to a consideration of the various sources of errors and imperfections. A crucial feature of our proposal is the fact that all nonleakage errors in the system localize. By nonleakage errors we refer to any decoherence which eventually returns the electrons back into the computational subspace-i.e., back into any state such that one electron is located in the orbital ground state of each dot. By localize we refer to the fact that the action of any decoherence map on the electrons is (mathematically) equivalent to a (different) decoherence map on some of the emitted photons; however, crucially the number of affected photons is at most the four photons emitted around the time the decoherence event occurs. This ensures that the final output state takes the form of an ideal cluster subject to localized random noise-a noise model for which fault-tolerant procedures are known to work. In particular we emphasize that this allows for production of photonic cluster states for arbitrarily longer times than the electron decoherence time scales might suggest.

The error localization might be seen in quite a general manner as follows. Consider the quantum circuit of Fig. 2 encoding the generic evolution. Let some decoherence occur which is described by a set of Kraus operators $\left\{K_{i}\right\}$ acting on the spin only. If we denote by $U$ the unitary evolution which corresponds in the figure to the circuit consisting of four photon emissions (i.e., two photons per dot and including the CZ gate acting between the dots) then an error and subsequent evolution take the generic form

$\rho_{i}^{\prime}=U\left(I \otimes I \otimes K_{i}\right)\left(\rho_{\text {spin }} \otimes|00\rangle\langle 00|\otimes| 00\rangle\langle 00|\right)\left(I \otimes I \otimes K_{i}^{\dagger}\right) U^{\dagger}$.

As mentioned above, it is a remarkably nice feature of this process that in fact we can find a Kraus operator $\tilde{K}_{i}$ acting now only on the four emitted photons, such that

$$
\rho_{i}^{\prime}=\left(\tilde{K}_{i} \otimes I\right) U\left(\rho_{\text {spin }} \otimes|00\rangle\langle 00|\otimes| 00\rangle\langle 00|\right) U^{\dagger}\left(I \otimes \tilde{K}_{i}^{\dagger}\right) .
$$


We now discuss some specific sources of error and their expected impact.

1. Imperfect $\mathrm{CZ}$ gate.-If we label $\Omega_{0}$ the Rabi frequency of the target transition from $|\downarrow \downarrow\rangle$, then the other transitions see a Rabi frequency of $\Omega_{1}=\Omega_{0} / \sqrt{3}$ and $\Omega_{2}=\sqrt{\frac{3}{2}} \Omega_{0}$, with a large detuning. As such, some population is transferred to those excited states and it is not returned via stimulated emission. Instead, the incoherent process of spontaneous emission redistributes that population. For simplicity we assume that the small population transferred is equal for the two unwanted transitions and that spontaneous emission equally redistributes it. The simplest way to express the Kraus operators $\left\{K_{j}\right\}$ describing the generalized quantum evolution in the two spin qubit subspace is by one nearly unitary, $\mathrm{CZ}$ operator:

$$
K_{0}=u_{1}|\uparrow \uparrow\rangle\left\langle\uparrow \uparrow|+| \psi^{-}\right\rangle\left\langle\psi^{-}\left|+u_{2}\right| \psi^{+}\right\rangle\left\langle\psi^{+}|-| \downarrow \downarrow\right\rangle\langle\downarrow \downarrow|,
$$

plus eight more operators describing the redistribution of the populations. For a pulse of a total duration of $40 \mathrm{ps}$ and for anisotropic exchange $\delta=0.5 \mathrm{meV}$, we have $\left|u_{1}\right| \simeq\left|u_{2}\right| \sim 0.99$. Then the remaining operators, $\left\{K_{1}, K_{2}, \ldots, K_{8}\right\}$, are $\frac{\sqrt{1-\left|u_{1}\right|^{2}}}{2}|k\rangle\langle 1|$ and $\frac{\sqrt{1-\left|u_{2}\right|^{2}}}{2}|k\rangle\langle 3|$, with $k=1,2,3,4$. Since the operator sum representation is not unique, we can find a different set of Kraus operators $\left\{M_{j}\right\}$ for which $M_{0}$ is proportional to the $\mathrm{CZ}$ gate. Setting $u_{1}=u_{2} \equiv u$, these are $M_{0}=\alpha \mathrm{CZ}, M_{1}=$ $e^{i \phi} \sqrt{1-\alpha^{2}} K_{0}-\frac{\alpha}{\sqrt{2}}\left(K_{1}+K_{2}\right)$, and $M_{2}=\frac{1}{\sqrt{2}}\left(K_{1}-K_{2}\right)$, with $\phi=\arctan \left(\frac{\operatorname{Im}(u)}{1-\operatorname{Re}(u)}\right)$. For $j=3, \ldots, 8, M_{j}=K_{j}$. The value of $\alpha$ is a measure of how close the operation is to a unitary CZ. For $u_{1}=u_{2}=0.99$ we find $\alpha=0.98$, with an error of $1-\alpha=0.02$. Physically we can therefore interpret the action of the gate as follows: With probability $\alpha^{2}$ we obtain a perfect CZ gate, with probability $\left(1-\alpha^{2}\right)$ we obtain some other type of evolution.

2. Unequal g factors.-In general, the two QDs comprising the QD molecule will have different $g$ factors, and therefore different precession frequencies. This means that we cannot get both spins to undergo a $R_{y}(\pi / 2)$ operation solely based on precession. One can correct for this mismatch by spin-echo-type control by applying to the fast spin at time $\tau=\pi\left(\omega_{s}^{-1}-\omega_{f}^{-1}\right) / 4$ a single qubit $\pi$ rotation about the optical axis to delay it $\left(\omega_{f}, \omega_{s}\right.$ are the fast and slow Zeeman splittings, respectively). These rotations are by design fast (in the picosecond regime) [14] and have been demonstrated experimentally [15].

3. Decay of the third electron into the lower orbital state of one of the QDs. - If the extra electron has decayed into the dot with the lowest single particle energy, where the hole is located by design, then one of the electrons in that dot recombines with the hole, resetting the dots to having one electron each. In the other case the extra electron decays into the QD with higher energy and recombination will occur of the electron in the low energy dot with the hole, leaving two electrons on one QD and the other dot uncharged. This state is energetically very unfavorable due to Coulomb repulsion, and so after a short time the electron tunnels to the lower energy QD, resetting the system to the computational basis. During this time the dots cannot emit any photons as all relevant transitions are at very different energies. Thus, this error will cause both quantum dots to stop emitting photons, and thus amounts to a detectable loss error on the cluster state.

4. Precession during $\mathrm{CZ}$ gate and dephasing of the electron.-These are both errors whose effect will be essentially the same as the corresponding ones in [5]. They result in localizable errors, which for suitable parameters can be extremely low [5].

In conclusion, we have developed a scheme for generation of a $2 \times N$ dimensional photonic cluster state based on coupled quantum dots. Analysis of the relevant errors shows our proposal to be robust and feasible with current state-of-the-art systems. This scheme can be generalized to the generation of a two-dimensional sheet by employing cavities and waveguides, with each quantum dot molecule situated in a cavity and each of its constituent QDs coupled by the waveguide to the adjacent cavity.

This work was supported in part by the Engineering and Physical Sciences Research Council and the U.S. Office of Naval Research and NSA/LPS.

[1] R. Raussendorf and H. J. Briegel, Phys. Rev. Lett. 86, 5188 (2001).

[2] R. Raussendorf et al., Ann. Phys. (N.Y.) 321, 2242 (2006).

[3] M. Varnava, D. E. Browne, and T. Rudolph, Phys. Rev. Lett. 97, 120501 (2006); 100, 060502 (2008).

[4] D. E. Browne and T. Rudolph, Phys. Rev. Lett. 95, 010501 (2005); Q. Zhang et al., Phys. Rev. A 77, 062316 (2008).

[5] N. H. Lindner and T. Rudolph, Phys. Rev. Lett. 103, 113602 (2009).

[6] S.E. Economou and T.L. Reinecke, Phys. Rev. B 78, 115306 (2008).

[7] A.E. B. Nielsen and K. Molmer, Phys. Rev. A 81, 043822 (2010); Y. Li, L. Aolita, and L.C. Kwek, arXiv:1003.1742v1.

[8] C. Santori et al., Phys. Rev. Lett. 86, 1502 (2001); A. Muller et al., Phys. Rev. Lett. 101, 027401 (2008).

[9] M. V. G. Dutt et al., Phys. Rev. Lett. 94, 227403 (2005).

[10] E. A. Stinaff et al., Science 311, 636 (2006).

[11] M. Bayer et al., Phys. Rev. B 65, 195315 (2002).

[12] M. Scheibner et al., Phys. Rev. B 75, 245318 (2007).

[13] D. Kim et al., arXiv:1007.3733v1.

[14] S. E. Economou et al., Phys. Rev. B 74, 205415 (2006).

[15] A. Greilich et al., Nature Phys. 5, 262 (2009); D. Press et al., Nature (London) 456, 218 (2008); J. Berezovsky et al., Science 320, 349 (2008) 